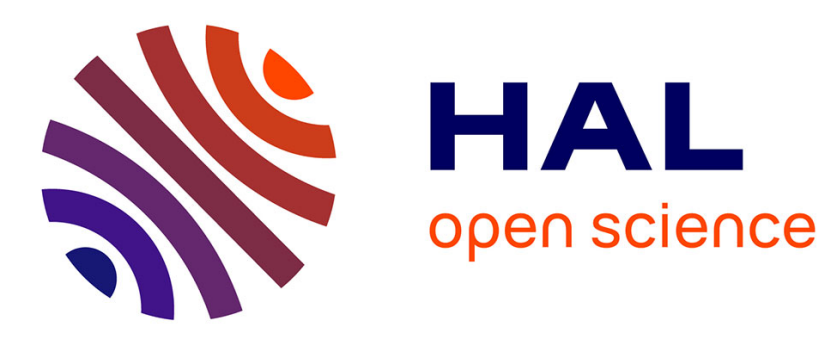

\title{
Results on the dimension spectrum for self-conformal measures
}

\author{
Michal Rams, Jacques Lévy Véhel
}

\section{To cite this version:}

Michal Rams, Jacques Lévy Véhel. Results on the dimension spectrum for self-conformal measures. Nonlinearity, 2007, 20 (4), pp.965-973. 10.1088/0951-7715/20/4/009 . hal-00539225

\section{HAL Id: hal-00539225 \\ https://hal.science/hal-00539225}

Submitted on 14 Mar 2011

HAL is a multi-disciplinary open access archive for the deposit and dissemination of scientific research documents, whether they are published or not. The documents may come from teaching and research institutions in France or abroad, or from public or private research centers.
L'archive ouverte pluridisciplinaire HAL, est destinée au dépôt et à la diffusion de documents scientifiques de niveau recherche, publiés ou non, émanant des établissements d'enseignement et de recherche français ou étrangers, des laboratoires publics ou privés. 


\title{
Results on the Dimension Spectrum for Self-conformal Measures
}

\author{
Michał Rams* \\ Institute of Mathematics, Polish Academy of Sciences \\ ul. Śniadeckich 8, 00-950 Warszawa, Poland \\ e-mail: rams@impan.gov.pl \\ Jacques Lévy Véhel \\ Projet Fractales \\ Inria, 78153 Le Chesnay Cedex, France \\ e-mail: jacques.levy-vehel@inria.fr
}

\begin{abstract}
We consider the natural measures associated with a family of conformal iterated function systems satisfying the transversality condition but no separation condition. We provide the exact value of their generalised Renyi dimensions $D_{q}$ for $q$ in a certain range.
\end{abstract}

\section{Introduction}

Let $V$ be an open and bounded subset of $\mathbb{R}^{d}$. For each parameter $t \in V$ we consider a conformal iterated function system $(\operatorname{IFS})\left(f_{i}(\cdot, t)\right)_{i=1}^{k}$ in $\mathbb{R}^{d}$ depending on $t$. We assume this dependence to be smooth (at least $C^{1+\beta}$ ). We denote by $\Lambda_{t}$ the limit set of the IFS, by $\nu_{t}$ its natural measure and by $s(t)$ the similarity dimension, i.e. the solution of the Bowen's equation

$$
P\left(s(t) \chi_{t}\right)=0
$$

where $\chi_{t}$ is the Lyapunov exponent of the IFS and $P$ denotes the topological pressure. It is well known that

$$
\operatorname{dim}_{H} \Lambda_{t} \leq s(t) .
$$

${ }^{*}$ supported by Polish KBN Grant No 2P0 3A 03425 
If, in addition, the IFS satisfies the Open Set Condition (OSC), it is a classical result (due to Hutchinson $[\mathrm{Hu}]$ and Manning and McCluskey $[\mathrm{MM}]$ ) that the Hausdorff dimension of the limit set equals $s(t)$. In this paper we assume that the transversality condition introduced by Pollicott and Simon [PoSi] (see definition 2.1) holds. We also assume that

$$
s(t)<d
$$

for all $t$.

A fine analysis of the properties of the natural measure $\nu_{t}$ of the IFS is provided by the computation of the so-called generalized dimensions or $D_{q}$ spectrum. These are computed as follows. Let, for $q \geq 0$ and $\varepsilon>0$,

$$
C_{q}\left(\nu_{t}, \varepsilon\right)=\int\left(\nu_{t}(B(x, \varepsilon))^{q-1} d \nu_{t}(x)\right.
$$

$(B(x, \varepsilon)$ denotes the closed ball of radius $\varepsilon$ centered at $x)$. For $q \neq 1$, one defines the lower and upper $q$-dimensions as:

$$
\begin{aligned}
& D_{q}^{-}\left(\nu_{t}\right)=\liminf _{\varepsilon \rightarrow 0} \frac{\log \left(C_{q}\left(\nu_{t}, \varepsilon\right)\right)}{(q-1) \log (\varepsilon)}, \\
& D_{q}^{+}\left(\nu_{t}\right)=\limsup _{\varepsilon \rightarrow 0} \frac{\log \left(C_{q}\left(\nu_{t}, \varepsilon\right)\right)}{(q-1) \log (\varepsilon)} .
\end{aligned}
$$

In case the limit exists, it is called the $q$-dimension of $\nu_{t}$, denoted $D_{q}\left(\nu_{t}\right)$. For $q=1$, one sets:

$$
\begin{aligned}
& D_{1}^{-}\left(\nu_{t}\right)=\liminf _{\varepsilon \rightarrow 0} \frac{\int \log \left(\nu_{t}(B(x, \varepsilon)) d \nu_{t}(x)\right.}{\log (\varepsilon)}, \\
& D_{1}^{+}\left(\nu_{t}\right)=\limsup _{\varepsilon \rightarrow 0} \frac{\int \log \left(\nu_{t}(B(x, \varepsilon)) d \nu_{t}(x)\right.}{\log (\varepsilon)} .
\end{aligned}
$$

Alternate, equivalent, definitions exist. See in particular [HK, JJ] for potential-theoretic ones.

Generalized dimensions are extensively used for the study of chaotic dynamical systems. In addition, the Legendre transform of the function $q \mapsto(q-1) D_{q}$ provides an upper bound to the so-called large deviation multifractal spectrum, with equality in a number of interesting cases (the simplest one being self-similar measures with the OSC, see, e.g. [LVT, LVV]). In such cases, one says that the weak multifractal formalism holds. Since we will use the definition later, we recall it now. For each positive integer $n$, we consider the partition of the support of the measure $\mu$ into dyadic cubes of 
sizes $2^{-n}$. For any $\varepsilon>0$ and any $\alpha \in \mathbb{R}$, let $N_{n}(\alpha, \varepsilon)$ denote the number of cubes with measure between $2^{-n(\alpha+\varepsilon)}$ and $2^{-n(\alpha-\varepsilon)}$. The large deviation multifractal spectrum is the function $([\mathrm{F}, \mathrm{LVV}, \mathrm{LVT}])$ :

$$
f(\alpha)=\lim _{\varepsilon \rightarrow 0} \limsup _{n \rightarrow \infty} \frac{\log \left(N_{n}(\alpha, \varepsilon)\right)}{n \log 2} .
$$

The existence of the $L^{q}$ dimension spectrum is known for the natural measure of the IFS, see [PeSo] for the linear case. This proof works (after minor changes) in nonlinear case as well, as shown in $[R]$ (the proof in $[R]$ is given for correlation dimension $D_{2}$ only, but the general case can be obtained in the same way). The result of Hunt and Kaloshin $[\mathrm{HK}]$ implies that $D_{q}=$ $s(t)$ for all $q \leq 2$ and for almost all $t \in V$ (we explain it in more details in the third section). They also give an example, showing that the constant 2 is sharp.

The purpose of this work is to prove the following result:

Theorem 1.1. If $s(t)<d / 2$ for all $t \in V$ then for almost all $t \in V$ the IFS satisfies the strong open set condition, hence

$$
D_{q}\left(\nu_{t}\right)=s(t)
$$

for all $q$.

If $s(t) \geq d / 2$ for all $t \in V$ then for almost all $t \in V$

$$
D_{q}\left(\nu_{t}\right)=s(t)
$$

for all $q \leq s(t) /(s(t)-d / 2)$ (if $s(t)>d / 2)$ or for all $q$ (if $s(t)=d / 2)$.

As an immediate corollary, we get:

Corollary 1.2. If $s(t) \geq d / 2$ for all $t \in V$ then for almost all $t \in V$ and for all $x \in \Lambda_{t}$ the pointwise dimension of $\nu_{t}$ at $x$ is at least $d / 2$. In case $s(t)=d / 2$ the pointwise dimension of $\nu_{t}$ equals $d / 2$ at every point (for almost all $t \in V$ ).

The results above hold for iterated function systems. They are not true for general projections of measures. For instance, we have the following example in $\mathbb{R}^{2}$ :

Example 1.3. For any $s<1$ there exists a transversal one-dimensional family of projections of $s$-dimensional self-similar measure $\mu_{s}$ such that

$$
D_{q}\left(\pi_{\theta}\left(\mu_{s}\right)\right)<s
$$

for all $q>(s+1) / s$ and for all values of parameter $\theta$. 
The paper is organized as follows: In the following section we introduce the notations and describe the local geometry of IFS. There, we also consider families of IFS. The proof of Theorem 1.1 is contained in the third section. Finally, in the fourth section, we present the construction of example 1.3.

\section{Local geometry of iterated function sys- tems}

For a conformal mapping from $\mathbb{R}^{d}$ into itself we will denote $f^{\prime}=|\operatorname{det} D f|^{1 / d}$.

An iterated function system is a finite family $\left(f_{i}\right)_{i=1}^{k}$ of contractive diffeomorphisms acting from $\mathbb{R}^{d}$ into itself. The limit set of IFS is the unique non-empty compact set $\Lambda$ satisfying the equation

$$
\Lambda=\bigcup f_{i}(\Lambda)
$$

The symbolic space of an IFS is defined as

$$
\Sigma=\{1, \ldots, k\}^{\mathbb{N}}
$$

its elements will be denoted by $\omega=\left(\omega_{1} \omega_{2} \ldots\right)$. The finite sequences of symbols $1, \ldots, k$ will be denoted by $\omega^{n}=\left(\omega_{1} \omega_{2} \ldots \omega_{n}\right)$.

On $\Sigma$ we define some mappings. The left shift $\sigma$ deletes the first digit of a sequence:

$$
\sigma\left(\omega_{1} \omega_{2} \ldots\right)=\left(\omega_{2} \omega_{3} \ldots\right) .
$$

The right shift $\sigma_{i}(i=1,2, \ldots, k)$ adds the symbol $i$ at the beginning of the sequence:

$$
\sigma_{i}\left(\omega_{1} \omega_{2} \ldots\right)=\left(i \omega_{1} \omega_{2} \ldots\right) .
$$

We write $f_{\omega^{n}}=f_{\omega_{1}} \circ \cdots \circ f_{\omega_{n}}, \sigma_{\omega^{n}}=\sigma_{\omega_{1}} \circ \cdots \circ \sigma_{\omega_{n}}, U_{\omega^{n}}=f_{\omega^{n}}(U)$ and $\Sigma_{\omega^{n}}=\sigma_{\omega^{n}}(\Sigma)$.

We define a projection from $\Sigma$ into $\mathbb{R}^{d}$ :

$$
\pi(\omega)=\lim _{n \rightarrow \infty} f_{\omega^{n}}(0)
$$

By $[\mathrm{Hu}], \Lambda=\pi(\Sigma)$. When $x=\pi(\omega)$, we will call $\omega$ the symbolic expansion of $x$ (it needs not be uniquely defined).

We demand that all the mappings $f_{i}$ are smooth (at least $C^{1+\beta}$ for some positive $\beta$ ) and conformal, at least in some neighborhood of $\Lambda$. The latter assumption is void in the one dimensional case but quite restricting (allowing only Möbius transformations) when $d \geq 3$. We denote by $U$ a neighborhood of $\Lambda$ on which these assumptions are satisfied and assume $U$ is bounded and 
open. As $f_{i}$ are $C^{1+\beta}$ contractions with universally bounded contraction ratio, all $f_{\omega^{n}}$ satisfy the following inequality (called Bounded Distortion Property, $\mathrm{BDP})$ :

$$
\exists L_{0} \forall_{x, y, z \in U} \forall_{\omega^{n}} L_{0}^{-1} \leq \frac{\left|f_{\omega^{n}}(x)-f_{\omega^{n}}(y)\right|}{|x-y| f_{\omega^{n}}^{\prime}(z)} \leq L_{0},
$$

see $[\mathrm{F}]$, chapter 4 .

We can freely demand that $U_{i} \subset U$ for all $i$ (for example, $U=B_{r}(\Lambda)$ will satisfy this). It follows that $U_{\omega^{n} i} \subset U_{\omega^{n}}$. The sets $U_{\omega^{n}}$ will be called cylinders.

We denote by $\lambda_{+}$and $\lambda_{-}$the maximum and minimum of local contraction ratios of all the mappings $f_{i}$ over all $x \in U$.

We will use sets $\Sigma_{\omega^{n}}=\sigma_{\omega^{n}}(\Sigma)$ and call them cylinders, too (in symbolic space). It is easy to see that $\pi\left(\Sigma_{\omega^{n}}\right) \subset U_{\omega^{n}}$; the cylinders $U_{\omega^{n}}$ and $\Sigma_{\omega^{n}}$ will be called dual.

We introduce a metric on $\Sigma$ given by $\rho(\omega, \tau)=\left|U_{\omega^{n}}\right|$, where $\omega_{i}=\tau_{i}$ for all $i \leq n$ but not for $i=n+1$. If $\omega_{1} \neq \tau_{1}$, then $\rho(\omega, \tau)=|U|$. This metric agrees with the product topology on $\Sigma$. It is easy to check that $\pi$ is a Lipschitz mapping (with Lipschitz constant 1) in the metric $\rho$.

We define a family of Hölder-continuous functionals on $\Sigma$ :

$$
\phi_{r}(\omega)=r \cdot \log \left(f_{\omega_{1}}^{\prime} \circ \pi \circ \sigma(\omega)\right) .
$$

There exists precisely one value $r=s$ for which the pressure (see, e.g., $[\mathrm{F}])$ of $\phi_{r}$ vanishes; it is called the similarity dimension of the IFS. Let $\mu$ be the Gibbs measure (on $\Sigma$ ) for $\phi_{s}$ (see, e.g., [BMP]) and denote by $\nu$ the projection of $\mu$ under $\pi$. We will call $\nu$ the natural measure of the IFS.

¿From the definition of Gibbs measure

$$
\mu\left(\Sigma_{\omega^{n}}\right) \approx \exp \left(\sum_{i=0}^{n-1} \phi_{s}\left(\sigma^{\circ i}(\omega)\right)\right) \approx\left(\frac{\left|U_{\omega^{n}}\right|}{|U|}\right)^{s} .
$$

Hence, $\mu$ is equivalent to the $s$-dimensional Hausdorff measure on $(\Sigma, \rho)$. In particular, neither the Hausdorff dimension of $\Lambda$ nor $D_{q}$ dimension of $\nu$ can be greater than $s$ (as the Lipschitz mappings cannot increase the Hausdorff or $D_{q}$ dimensions).

For all $l \leq|U|$ we will define $Z_{l}$ as a family of all cylinders $U_{\omega^{n}}$ such that

$$
\left|U_{\omega^{n}}\right| \leq l<\left|U_{\omega^{n-1}}\right|
$$

The cylinders in $Z_{l}$ have diameter between $l$ and $\lambda_{-} l$. 
By (2.3), the dual cylinders to those in $Z_{l}$ have measure $\mu$ between $L_{4}^{-1}(l /|U|)^{s}$ and $L_{4}(l /|U|)^{s}$ for some $L_{4}$ for all $l$. Hence $Z_{l}$ has between $L_{4}^{-1}(l /|U|)^{-s}$ and $L_{4}(l /|U|)^{-s}$ elements.

We define the intersection numbers for cylinders from $Z_{l}$ :

$$
A_{l}=\sharp\left\{\left(U_{\omega^{n}}, U_{\kappa^{m}}\right) \in Z_{l} \times Z_{l}: U_{\omega^{n}} \cap U_{\kappa^{m}} \neq \emptyset, \omega_{1} \neq \kappa_{1}\right\} .
$$

Let

$$
l_{i}=|U| \lambda_{+}^{i}
$$

For such a sequence every cylinder $U_{\omega^{n}}$ belongs to at least one and at most some $L_{7}$ of Moran covers $Z_{l_{i}}$, where $L_{7}$ does not depend on $\omega^{n}$.

We will now consider not a single IFS but a $d$-dimensional family of IFS, acting on $\mathbb{R}^{d}$. We will use $t=\left(t_{1}, \ldots, t_{d}\right)$ as a parameter and write the dependence on $t$ explicitly; for example, the limit set will be denoted as $\Lambda_{t}$. The set of parameters $\bar{V}$ is assumed to be the closure of a bounded open subset of $\mathbb{R}^{d}$. We assume the contractions $f_{i}(x ; t)$ to be $C^{1+\beta}$ for $(x, t) \in$ $U \times \bar{V}$, that is we want all the derivatives $\partial f_{i} / \partial x_{j}$ and $\partial f_{i} / \partial t_{j}$ to be $C^{\beta}$ with respect to both $x$ and $t$.

Denote $h_{\omega, \kappa}(t)=\pi_{t}(\omega)-\pi_{t}(\kappa)$. The following definition was first introduced (in a one-dimensional situation) in [PoSi].

Definition 2.1. The family of IFS satisfies the transversality condition if there exists a constant $L_{5}$ such that for any two sequences $\omega, \kappa \in \Sigma$ with $\omega_{1} \neq \kappa_{1}$, if $\left|h_{\omega, \kappa}(u)\right|<L_{5}$ then $\left|\operatorname{det} D_{t} h_{\omega, \kappa}(t)\right|_{\mid t=u}>L_{5}$.

The following lemma wasn't explicitly stated in $[R]$ but it was proved (under the transversality condition) in the course of proof of Theorem 1.1

Lemma 2.2. For any $\varepsilon>0$ and Lebesgue-almost any $t \in V$ there exists $L_{6}(t)$ such that for all $l \leq|U|$

$$
A_{l}(t) \leq L_{6} l^{-2(1+\varepsilon) s(t)+d}
$$

\section{Proof of the Theorem 1.1}

As mentioned above, $D_{q}\left(\nu_{t}\right) \leq s(t)$ for all $q$. The result of Hunt and Kaloshin implies that for almost all $t \in V$ the equality holds for all $q$ between 1 and 2. By monotonicity of $D_{q}$, the equality must hold for all $q \leq 1$ as well. In what follows we are only interested in $q$ greater than 2 .

The first part of Theorem 1.1 is easy to prove. The strong open set condition is equivalent to the bijectivity of $\pi$. Assume $\pi_{t}(\omega)=\pi_{t}(\tau)$ for 
some $\omega \neq \tau$. We may freely assume $\omega$ and $\tau$ have different first symbols, otherwise we have $\pi_{t}(\sigma \omega)=\pi_{t}(\sigma \tau)$ and we repeat this as long as the first digits are not different. Hence, $U_{\omega^{m}}(t)$ and $U_{\tau^{n}}(t)$ intersect for all $m, n$. In particular, $A_{l_{i}}(t)>0$ for all $i$. This can only happen for a zero measure set of parameters by Lemma 2.2. A more detailed analysis, based not on Lemma 2.2 but on Proposition 4.6 from [R], shows that this set of parameters has its upper box dimension not greater than $2 \sup s(t)$.

Assume now that $s(t)>d / 2$ and the assertion of Lemma 2.2 is satisfied for $t$. As $t$ is henceforth fixed, we denote $s=s(t)$ etc.

The proof works as follows. We are going to estimate from above the multifractal spectrum $f(\alpha)$ by estimating maximal possible number of places where many cylinder sets $U_{\omega^{n}}$ meet. The direct estimation by means of Lemma 2.2 is not enough because it gives us only the number of pairs of intersecting cylinder sets with different first digits of their symbolic expansion (i.e. $U_{\omega^{n}}$ intersects $U_{\tau^{m}}$ and $\omega_{1} \neq \tau_{1}$ ). We will perform a more delicate estimation using the fact that if $U_{\omega^{n}}$ and $U_{\tau^{m}}$ intersect each other and $\omega_{1}=\tau_{1}$ then the cylinder sets $U_{\sigma \omega^{n}}$ and $U_{\sigma \tau^{m}}$ must intersect each other as well (thus we get to use Lemma 2.2 on many different scales).

Let $\delta$ be a small positive constant. Given $l$ and $N$, consider a set of all points belonging to at least $N$ sets $U_{\omega^{n}} \in Z_{l}$ and let $M(l, N)$ be its maximal $\lambda_{-}^{-1} l$-distanced subset, i.e. points from $M(l, N)$ are in distance greater than $\lambda_{-}^{-1} l$ from each other, belong to at least $N$ sets $U_{\omega^{n}} \in Z_{l}$ and no point with those properties can be further added to $M(l, N)$. Note that two different points from $M(l, N)$ cannot belong to the same $U_{\omega^{n}} \in Z_{l}$.

Given a finite word $\omega^{n}$, let $P\left(l, N, \omega^{n}\right)$ be the subset of $M(l, N)$ consisting of the points that belong to at least $N(1-\delta)^{n}$ sets $U_{\tau^{m}} \in Z_{l}$ with the symbolic expansions beginning with $\omega^{n}$, ie. $\tau^{m}=\omega^{n} \eta^{m-n}$. Let also

$$
Q\left(l, N, \omega^{n}\right)=P\left(l, N, \omega^{n}\right) \backslash \bigcup_{i=1}^{k} P\left(l, N, \omega^{n} i\right)
$$

Clearly,

$$
M(l, N)=\bigcup_{\omega^{n}} Q\left(l, N, \omega^{n}\right)
$$

where the union goes over all finite sequences with $\left|U_{\omega^{n}}\right|>l \lambda_{-}$.

\section{Lemma 3.1.}

$$
\sharp\left(Q\left(l, N, \omega^{n}\right)\right) \leq L_{1} l^{-2 s(1+\varepsilon)+d}\left(\frac{|U|}{\left|U_{\omega^{n}}\right|}\right)^{-2 s(1+\varepsilon)+d}(1-\delta)^{-2 n} \delta^{-2} N^{-2}
$$


Proof. Let $x \in Q\left(l, N, \omega^{n}\right)$. The Dirichlet principle easily shows that for any $x$ there are two different symbols $i, j$ such that among the sets $U_{\tau^{m}} \in Z_{l}$ that contain $x$ there are at least $N(1-\delta)^{n} \delta / k$ such that their symbolic expansions begin with $\omega^{n} i$ and the same number beginning with $\omega^{n} j$.

Hence, there are at least $N^{2}(1-\delta)^{2 n} \delta^{2} k^{-2}$ pairs of sets $\left(U_{\tau^{m+n+1}}, U_{\eta^{r+n+1}}\right)$ such that

i) $x \in U_{\tau^{m+n+1}} \cap U_{\eta^{r+n+1}}$,

ii) $\tau^{m+n+1}=\omega^{n} i(x) \phi^{m}$,

iii) $\eta^{r+n+1}=\omega^{n} j(x) \xi^{r}$,

iv) both $\tau^{m+n+1}$ and $\eta^{r+n+1}$ do not appear again for any $y \in Q\left(l, N, \omega^{n}\right)$.

Hence, we have at least $N^{2}(1-\delta)^{2 n} \delta^{2} k^{-2} \sharp Q\left(l, N, \omega^{n}\right)$ different pairs for all points in $Q\left(l, N, \omega^{n}\right)$.

The image under $f_{\omega^{n}}^{-1}$ of any such pair is the pair of intersecting sets $\left(U_{i \phi^{m}}, U_{j \xi^{r}}\right)$. Those sets have diameter between $l L_{0}|U| /\left|U_{\omega^{n}}\right|$ and $l \lambda_{-} L_{0}^{-1}|U| /\left|U_{\omega^{n}}\right|$ by $(2.2)$. For any such pair let $g\left(\left(U_{i \phi^{m}}, U_{j \xi^{r}}\right)\right)=\left(U_{i \phi^{m}}, U_{j \xi^{r} 0}\right)$ be the pair of their ancestors, belonging to $Z_{l L_{0}|U| /\left|U_{\omega} n\right|}$.

The mapping $g$ is uniquely defined. In addition

$$
\lambda_{+}^{\max \left(m-m_{0}, r-r_{0}\right)} \geq \lambda_{-} L_{0}^{-2},
$$

hence $g$ is at most a $K$ to 1 mapping for some uniformly bounded $K$. This proves that there are at least $N^{2}(1-\delta)^{2 n} \delta^{2} k^{-2} K^{-1} \sharp Q\left(l, N, \omega^{n}\right)$ different pairs of the form $g\left(\left(U_{i \phi^{m}}, U_{j \xi^{r}}\right)\right)$. Those are pairs of intersecting sets from $Z_{l L_{0}|U| /\left|U_{\omega^{n} \mid}\right|}$ with different first symbols, hence the number of such pairs cannot exceed $A_{l L_{0}|U| / \mid U_{\omega} n}$. The assertion then follows by Lemma 2.2.

Proposition 3.2. For $\delta$ small enough,

$$
\sharp M(l, N) \leq L_{2} \delta^{-2 s /(2 s-d)} l^{-s(1+2 s \varepsilon /(2 s-d))} N^{-2 s /(2 s-d)}
$$

where $L_{2}$ depends neither on $l$ nor on $N$.

Proof. By (3.1)

$$
\sharp M(l, N) \leq \sum_{\omega^{n}} \sharp Q\left(l, N, \omega^{n}\right) \leq \sum_{i} \sum_{U_{\omega^{n}} \in Z_{l_{i}}} \sharp Q\left(l, N, \omega^{n}\right)
$$

As

$$
n \leq \frac{\log \left(|U| / l_{i}\right)-\log \lambda_{-}}{-\log \lambda_{+}}
$$


we can estimate

$$
(1-\delta)^{n} \geq\left(\frac{l_{i} \lambda_{-}}{|U|}\right)^{s \varepsilon}
$$

for $\delta$ small enough. Hence, as the summands in (3.2) are nonnegative integers, they must be zero for

$$
\left(\frac{|U|}{l_{i}}\right)^{2 s-d}>L_{1} \lambda_{-}^{-2 s(1+2 \varepsilon)+d} l^{-2 s(1+\varepsilon)+d} \delta^{-2} N^{-2} .
$$

Let $I$ be the maximal $i$ that do not satisfy (3.4). We have

$$
\begin{gathered}
\sharp M(l, N) \leq \sum_{i \leq I} \sharp Z_{l_{i}} L_{1} \lambda_{-}^{-2 s(1+2 \varepsilon)+d} l^{-2 s(1+\varepsilon)+d}\left(\frac{|U|}{l_{i}}\right)^{-2 s+d} \delta^{-2} N^{-2} \leq \\
\leq \sum_{i \leq I} L_{1} \lambda_{-}^{-2 s(1+2 \varepsilon)+d} L_{4} l^{-2 s(1+\varepsilon)+d}\left(\frac{|U|}{l_{i}}\right)^{d-s} \delta^{-2} N^{-2}
\end{gathered}
$$

Substituting (2.4) we get an increasing exponential series. Its sum is (up to a constant) equal to the greatest element, which gives the assertion.

Note that $\delta$ depends on $\varepsilon$ but nothing else. Substituting $N=l^{-s+\alpha}$ for $d / 2 \leq \alpha<s$, we get

$$
\sharp M\left(l, l^{-s+\alpha}\right) \leq c(\varepsilon) l^{s(2 \alpha-d) /(2 s-d)-O(\varepsilon)}
$$

The estimation for $\sharp M(l, N)$ obtained here will allow us to obtain an estimation on the multifractal spectrum of $\nu$.

\section{Proposition 3.3.}

$$
f(\alpha) \leq \limsup _{l \rightarrow 0} \frac{\log \sharp M\left(l, l^{-s+\alpha}\right)}{-\log l}
$$

Proof. We fix $l$ for a moment. The natural measure $\nu$ is a projection of measure $\mu$ defined on $\Sigma$, which in turn can be presented as union of $\Sigma_{\omega^{n}}$ over $\left\{\omega^{n} ; U_{\omega^{n}} \in Z_{l}\right\}$. Hence, $\nu$ is a sum of approximately $l^{-s}$ separate measures $\pi_{*}\left(\chi\left(\Sigma_{\omega^{n}}\right) \cdot \mu\right)$, each supported on a $d$-dimensional approximate ball of diameter between $\lambda_{-} l$ and $l$ (by definition of $Z_{l}$ ) and each of norm between $L_{4}^{-1}(l /|U|)^{s}$ and $L_{4}(l /|U|)^{s}$ (by $(2.3)$ ). Given any set $Z \subset \mathbb{R}^{d}$ the following estimation holds:

$L_{4}^{-1}(l /|U|)^{s} \sharp\left\{U_{\omega^{n}} \in Z_{l} ; U_{\omega^{n}} \subset Z\right\} \leq \nu(Z) \leq L_{4}(l /|U|)^{s} \sharp\left\{U_{\omega^{n}} \in Z_{l} ; U_{\omega^{n}} \cap Z \neq \emptyset\right\}$ 
We will make two claims. Firstly, for any $x \in M(l, N)$ we claim that $\nu\left(B_{l}(x)\right) \geq N L_{4}^{-1}(l /|U|)^{s}$. Let us denote

$$
L_{10}=\inf _{\omega^{n}} \frac{\operatorname{vol}\left(U_{\omega^{n}}\right)}{\left|U_{\omega^{n}}\right|^{d}}
$$

(this number isn't zero because $U_{\omega^{n}}=f_{\omega^{n}}(U)$ and maps $f_{\omega^{n}}$ have uniformly bounded distortion). Our second claim is that for any $x \in \mathbb{R}^{d}$ if $\nu\left(B_{l}(x)\right) \geq N L_{4} L_{10}^{-1}(l /|U|)^{s} 4^{d} \lambda_{-}^{-d}$ then $\operatorname{dist}(x, M(l, N)) \leq\left(2+\lambda_{-}^{-1}\right) l$. Together those claims mean that $\sharp M(l, N)$ is (up to a constant) a number of separate balls of radius $l$ and measure at least $c N l^{s}$. The assertion then follows from the definition of multifractal spectrum.

The first claim follows directly from (3.6) because $B_{l}(x)$ must contain all $U_{\omega^{n}} \in Z_{l}$ that contain $x$. To prove the second claim, consider a ball $B_{l}(x)$ of big measure:

$$
\nu\left(B_{l}(x) \geq N L_{4} L_{10}^{-1}(l /|U|)^{s} 4^{d} \lambda_{-}^{-d}\right.
$$

By (3.6), $B_{l}(x)$ intersects (hence, $B_{2 l}(x)$ contains) at least $N L_{10}^{-1} 4^{d} \lambda_{-}^{-d}$ sets from $Z_{l}$. The sum of volumes of those sets is not smaller than $4^{d} \mathrm{Nl}^{d}$, i.e. more than $N$ times greater than the volume of $B_{2 l}(x)$. Hence, there is a point $y \in B_{2 l}(x)$ belonging to at least $N$ of them. Either $y$ belongs to $M(l, N)$ or there is another point in $M(l, N)$ in distance smaller than $\lambda_{-}^{-1} l$ from $y$ (hence in distance smaller than $\left(2+\lambda_{-}^{-1}\right) l$ from $x$ ), so the second claim is proved.

Proposition 3.3 together with (3.5) gives us the upper bound for the multifractal spectrum:

$$
f(\alpha) \leq \frac{s(2 \alpha-d)}{2 s-d}
$$

for $\alpha<s$. Using Legendre transform we get

$$
D_{q}(\nu)=s
$$

for $q \leq s /(s-d / 2)$ and we are done.

The last case that remains to be considered is $s(t)=d / 2$. Proposition 3.2 isn't working in this case but Lemma 3.1, Proposition 3.3 and formulas (3.2), (3.3) are. Substituting the formulas into the assertion of Lemma 3.1 and using inequality $l_{i} \geq l$ we get the estimation of the form

$$
\sharp\left(Q\left(l, N, \omega^{n}\right)\right) \leq c l^{-3 d \varepsilon / 2} \delta^{-2} N^{-2}
$$

By (3.4), for $M(l, N)$ to be nonempty $Q\left(l, N, \omega^{n}\right)$ must be nonempty as well for at least some $\omega^{n}$ and it is only possible when

$$
N \leq c^{1 / 2} \delta^{-1} l^{-3 d \varepsilon / 4}
$$


It implies that the multifractal spectrum $f(\alpha)$ equals $-\infty$ for all $\alpha<$ $s-3 d \varepsilon / 4$. As $\varepsilon$ can be chosen arbitrarily small, the graph of $f$ must actually contain only one point $(d / 2, d / 2)$ and the assertion follows.

\section{Example}

The example we present can be seen as a generalization of the Hunt and Kaloshin example.

Let $\lambda=2^{-1 / s}$. Let $I_{0}=[0,1] \times\{0\}$ be an interval of length 1 . We will construct inductively a family of unions of intervals in the following way. For every interval $I_{n}^{(i)} \subset I_{n}$ we first remove from $I_{n}^{(i)}$ the middle part of length $\left|I_{n}^{(i)}\right|(1-2 \lambda)$ and then rotate one of the resulting intervals (clockwise) by an angle $2^{-n-1} \pi$. The union of all the resulting intervals will be denoted $I_{n+1}$. It is easy to see that $I_{n}$ will be an union of $2^{n}$ intervals of length $\lambda^{n}$ each and their directions are of the form $i 2^{-n} \pi$ (see figure).

For any $I_{n}^{(i)}$, let $J_{n}^{(i)}$ be the ball of diameter $I_{n}^{(i)}$. Let $K_{n}^{(i)}$ be the intersection of $J_{n}^{(i)}$ with the $2 \lambda^{n} 2^{-n}$-neighborhood of $I_{n}^{(i)}$. The sets $K_{n}^{(\cdot)}$ are pairwise disjoint. Moreover, if $I_{n+1}^{\left(j_{1}\right)}$ and $I_{n+1}^{\left(j_{2}\right)}$ are the two intervals obtained from $I_{n}^{(i)}$ by the induction procedure, $K_{n+1}^{\left(j_{1}\right)}$ and $K_{n+1}^{\left(j_{2}\right)}$ are contained in $K_{n}^{(i)}$. Hence, $K_{n}=\bigcup_{j} K_{n}^{(j)}$ form a decreasing family of compact sets. We denote the limit set by $K$. We distribute a measure $\nu$ on $K$ in such a way that $\nu\left(K_{n}^{(j)}\right)=2^{-n}$ for all $j$. 

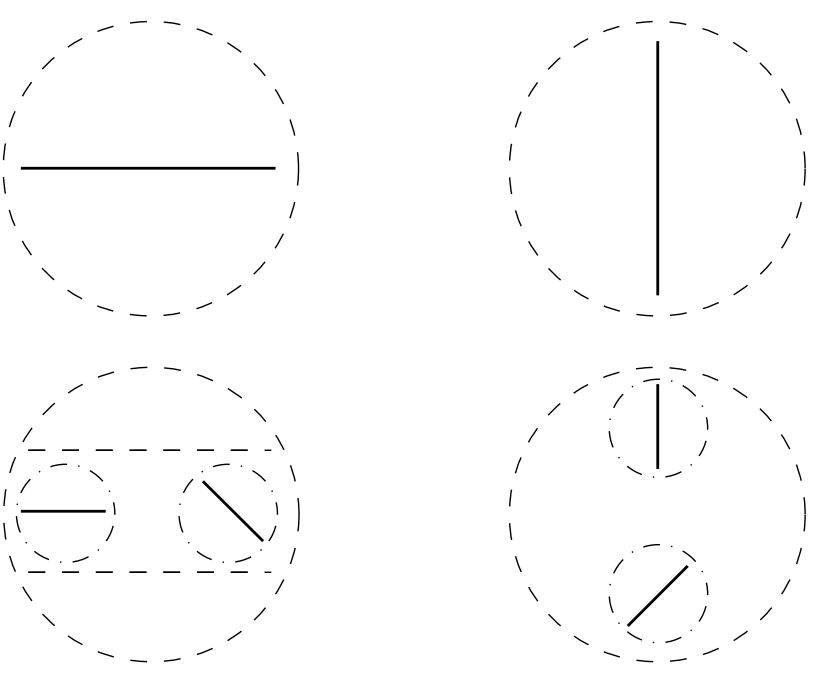

The sets $I_{0}$ (up, solid line), $I_{1}$ (middle, solid lines), $I_{2}$ (bottom, solid lines).

Consider a family of all orthogonal projections of $\nu$ on lines in $\mathbb{R}^{2}$. We denote the projection in direction $\theta$ by $\pi_{\theta}$ and let $\nu_{\theta}=\pi_{\theta *} \nu$. For any $n$ and any direction $\theta$ there is some $i$ for which the angle between $\theta$ and the direction of $I_{n}^{(i)}$ is smaller than $2^{-n} \pi$. Hence, the projection of $K_{n}^{(i)}$ in direction $\theta$ is an interval of length not greater than $(2+\pi) \lambda^{n} 2^{-n}$. At the same time, the measure $\nu_{\theta}$ of this interval is at least $2^{-n}$. Letting $n$ tend to $\infty$, we will get a point for which the pointwise dimension of $\nu_{\theta}$ is at most $-\log 2 / \log (\lambda / 2)=$ $s /(1+s)$. Hence, $D_{q}\left(\mu_{\theta}\right)<s$ for all $q>(s+1) / s$.

Consider now the standard $s$-dimensional self-similar measure $\mu$, ie. normalized $s$-dimensional Hausdorff measure on middle- $\gamma$ Cantor set, $\gamma=(1-$ $2 \lambda)$. The measure $\mu$ is bi-Lipschitz equivalent to $\nu$. Let us denote the conjugacy map by $h$. As the orthogonal projections form a transversal family and bi-Lipschitz conjugacy preserves transversality, the maps $h_{\theta}=\pi_{\theta} \circ h$ form a transversal family of projections of a self-similar measure but do not preserve its $D_{q}$ dimension for $q>(s+1) / s$. 


\section{References}

[BMP] G. Brown, G. Michon, and J. Peyrière. On the multifractal analysis of measures. J. Statist. Phys., 66(3-4):775-790, 1992.

[F] K.J. Falconer Techniques in Fractal Geometry, John Wiley 65 Sons, Chichester, (1997).

[HK] B. R. Hunt, V. Kaloshin, How projections affect the dimension spectrum of measures, Nonlinearity 10 (1997), 1031-1046.

[Hu] J. E. Hutchinson, Fractals and self-similarity, Indiana Univ. Math. J. 30 (1981), 713-747.

[JJ] E. Jarvenpaa, M. Jarvenpaa Linear mappings and generalized upper spectrum for dimensions, Nonlinearity 12 (1999), 475-493.

[LVT] J. Lévy Véhel, C. Tricot On various multifractal spectra Fractal Geometry and Stochastics III, Progress in Probability, C. Bandt, U. Mosco and M. Zhle (Eds),Birkhuser Verlag 57 (2004), 23-42.

[LVV] J. Lévy Véhel, R. Vojak Multifractal Analysis of Choquet Capacities: Preliminary Results, Advances in Applied Mathematics 20 (1998), $1-43$.

[MM] A. Manning, H. McCluskey, Hausdorff dimension for horseshoes, Erg. Th. and Dyn. Sys. 3 (1983), 251-260.

[PeSo] Y. Peres, B. Solomyak, Existence of $L^{q}$ dimensions and entropy dimension for self-conformal measures, Indiana Univ. Math. J. 49 (2000), 1603-1621.

[PoSi] M. Pollicott, K. Simon, The Hausdorff dimension of $\lambda$-expansions with deleted digits, Trans. of the Amer. Math. Soc. 347 (1995), 967-983.

[R] M. Rams, Packing dimension estimation for exceptional parameters, Israel Math. J. 130 (2002), 125-144. 